PESQUIMAT, Revista de la F.C.M. de la

Universidad Nacional Mayor de San Marcos

Vol. XIV N², pp. 97-108, Lima - Perú, Agosto 2011

\title{
ÍNDICE DE DESARROLLO HUMANO MEDIANTE METANÁLISIS BAYESIANO Y RAZÓN DE MORTALIDAD MATERNA CON METANÁLISIS CLÁSICO
}

\author{
Erwin Kraenau* \& María Ponce**
}

Resumen: El objetivo de la presente investigación fue sintetizar cuantitativamente la información de la Razón de la Mortalidad Materna (RMM) para los años 1990, 1995, 2000, 2005 y 2008 aplicando el Metanálisis Clásico; y el Índice de Desarrollo Humano (IDH) para el año 2010 aplicando el Metanálisis Bayesiano, combinando las distintas investigaciones desarrolladas en cada país; los resultados obtenidos se muestran para las regiones de Sudamérica y Latinoamérica (los datos utilizados fueros publicados por la Organización Mundial de la Salud y el Programa de Naciones Unidas para el Desarrollo). Los resultados para el IDH de Sudamérica y Latinoamérica fueron 0.6971 y 0.6719 , respectivamente. Adicionalmente, los RMM para Sudamérica los años 1990, 1995, 2000, 2005 y 2008 fueron 123, 101, 90.1, 82.1 y 80.7, respectivamente, y las RMM para Latinoamérica fueron 117, 105, 90.179 .1 y 75.5 , respectivamente. La heterogeneidad de los estudios individuales (por países) es inherente a estas mediciones, la cual se detectó analíticamente utilizando la prueba Q y visualmente mediante el Metaplot.

Palabras clave: RMM, IDH, Metanálisis Clásico, Metanálisis Bayesiano, Metaplot.

\section{HUMAN DEVELOPMENT INDEX USING BAYESIAN META-ANALYSIS AND MATERNAL MORTALITY RATIO WITH CLASSIC META-ANALYSIS}

\begin{abstract}
The objective of this research was to quantitatively synthesize the information from the Maternal Mortality Ratio (MMR) for the years 1990, 1995, 2000, 2005 and 2008 using the Classic Meta-analysis, and the Human Development Index (HDI) for the year 2010 using the Bayesian Meta-analysis combining the various investigations carried out in each country, the results obtained are shown for the regions of South America and Latin America (data used were published by the World Health Organization and United Nations Program for Development). The results for the HDI of South America and Latin America were 0.6971 and 0.6719, respectively. Additionally, the MMR for South America the 1990, 1995, 2000, 2005 and 2008 were 123, 101, 90.1, 82.1 and 80.7, respectively, and MMR for Latin America at 117, 105, 90.179 .1 and 75.5, respectively. The heterogeneity of individual studies (by countries) is inherent in these measurements, which was detected using the $\mathrm{Q}$ test analytically and visually by Metaplot.
\end{abstract}

Key words: MMR, HDI, Classic Meta-analysis, Bayesian Meta-analysis, Metaplot.

\section{Introducción}

La mejora de la salud materna y la reducción de la mortalidad materna han sido puntos clave de varias cumbres y conferencias internacionales desde finales de los ochenta, y también de la Cumbre del Milenio del año 2000. Uno de los ocho Objetivos de Desarrollo del Milenio (ODM) adoptados en la Cumbre del Milenio es mejorar la salud materna, dentro del marco de seguimiento de los ODM, la comunidad internacional se comprometió a reducir entre 1990 y 2015 la RMM en tres cuartos.

La RMM se define como el número de defunciones maternas durante un mismo período de tiempo en una

\footnotetext{
${ }^{*}$ UNMSMS, Facultad de Ciencias Matemáticas, e-mail: stoned@ec-red.com

${ }^{* *}$ UNMSM, Facultad de Ciencias Matemáticas, e-mail: mepaunmsm@yahoo.es
} 
población, dividida por cada 100,000 nacidos vivos en el mismo período; representa el riesgo de defunción materna en relación con el número de nacidos vivos.

El desarrollo humano, según el Programa de las Naciones Unidas para el Desarrollo (PNUD), es aquel que sitúa a las personas en el centro del desarrollo, trata de la promoción del desarrollo potencial de las personas, del aumento de sus posibilidades y del disfrute de la libertad para vivir la vida que valoran.

El desarrollo humano es el proceso por el que una sociedad mejora las condiciones de vida de sus ciudadanos a través de un incremento de los bienes con los que puede cubrir sus necesidades básicas y complementarias, y de la creación de un entorno en el que se respeten los derechos humanos de todos. El desarrollo humano podría definirse también como una forma de medir la calidad de vida del ser humano en el medio en que se desenvuelve, y es una variable fundamental para la calificación de un país o región. La información proporcionada por la OMS y el PNUD, es el resultado individual de la investigación desarrollada en cada país, dentro de su contexto, tiempo, recursos y metodología aplicada. Nos preguntamos: ¿Se pueden construir estimadores combinados (pooled) del IDH y RMM a partir de las investigaciones individuales, que describa algún aspecto de la realidad Sudamericana y Latinoamericana? La respuesta a la pregunta anterior fue respondida con la aplicación del metánalisis, considerando que los datos disponibles de la RMM para cada país está elaborada con distintos diseños muestrales y considerando que para el IDH sólo se dispone de estimaciones puntuales.

Los objetivos al aplicar esta metodología estadística propuesta son:

1. Mostrar la importancia de la aplicación del Metanálisis Clásico y Bayesiano como alternativa para sintetizar la información de la RMM y del IDH para la región de Sudamérica y Latinoamérica.

2. Enriquecer los resultados que muestra la OMS y el PNUD sobre estos indicadores.

3. Motivar a los investigadores a utilizar esta metodología, que permite ahorrar tiempo y recursos económicos, sin disminuir la confiabilidad de los resultados.

El procesamiento de los datos se hizo a través de la ejecución de los códigos fuentes diseñados para MATLAB versión R2010a y R Project versión 2.13.1.

\section{Metodología}

\subsection{Metanálisis Clásico}

El término metanálisis se refiere al análisis estadístico de una serie de resultados obtenidos individualmente con la finalidad de integrarlos, produciendo conclusiones estadísticas basadas en la revisión sistemática, principalmente se utiliza para calcular un valor medio global de la medida de interés, o para analizar la heterogeneidad de los diferentes resultados individuales. Es decir los objetivos del metanálisis son: la estimación de un efecto global y la cuantificación de la heterogeneidad de los resultados.

Aunque existen diferentes técnicas estadísticas para combinar resultados, hay tres fundamentales, que se conocen con el nombre modelo de efectos fijos, modelo de efectos aleatorios y por último el modelo Bayesiano.

\section{Modelo de efectos fijos}

El método más antiguo y popular para combinar resultados de estudios individuales siguiendo un modelo de efectos fijos se conoce como método ponderado por la inversa de la varianza (inverse variance weighted method), y fue desarrollado en 1930 por Birge y luego por Cochran [?]. En este método cada estudio individual contribuye a la estimación global de forma proporcional a la precisión de cada resultado, donde el peso está dado por la inversa de la varianza, por ser la varianza una medida de dispersión y por lo tanto su inversa una medida de precisión.

Para formular este modelo, se asume que se tiene $k$ resultados de estudios individuales, donde cada $y_{i}$ es 
la medición del efecto individual en el $i$-ésimo estudio, para $i=1,2, \ldots, k$. Se considera que existe un efecto global fijo $\mu$ que se estima como:

$$
\bar{y}=\frac{\sum_{i=1}^{k} w_{i} y_{i}}{\sum_{i=1}^{k} w_{i}}
$$

donde los pesos $w_{i}$ de (??) son obtenidos por:

$$
w_{i}=\frac{1}{s_{i}^{2}}
$$

siendo $s_{i}^{2}$ la varianza observada de la medida del efecto en el estudio $i$-ésimo, cuya expresión analítica o aproximada depende del tipo de medida utilizada (razón de ventajas, riesgo relativo, media, etc.). La estimación de la varianza de $\bar{y}$ se obtiene mediante la siguiente expresión:

$$
V(\bar{y})=\frac{1}{\sum_{i=1}^{k} w_{i}}
$$

Si se puede asumir que $\bar{y}$ sigue una distribución normal se podrán obtener intervalos de confianza para el efecto global en función de la raíz cuadrada de la expresión (??).

Algunas medidas requieren una transformación previa, tomar logaritmos, antes de que puedan ser combinadas en un metanálisis, con el fin de que se aproximen a una distribución normal. Es lo que ocurre con la razón de ventajas y el riesgo relativo, entre otros, por lo que frecuentemente se trabaja con sus transformaciones logarítmicas [?].

\section{Modelo de efectos aleatorios}

Si los resultados de los estudios incluidos en el análisis constituyen una muestra aleatoria del universo de estudios posibles, se considera más apropiado utilizar el modelo de efectos aleatorios, donde el efecto $y_{i}$ en cada uno de los estudios se expresa mediante la suma de dos componentes:

$$
y_{i}=\theta_{i}+e_{i}
$$

donde $e_{i}$ es el error con el que $y_{i}$ estima el verdadero efecto $\theta_{i}$, por lo que la varianza de (??) viene dada por la expresión:

$$
V\left(y_{i}\right)=\tau_{\theta}^{2}+\nu_{i}
$$

siendo $\nu_{i}$ la varianza debida al error de muestreo en el $i$-ésimo estudio y $\tau_{\theta}^{2}$ corresponde a la varianza entre estudios.

La estimación de la varianza debida a la variación entre estudios, se hace a partir de las siguientes expresiones:

$$
\begin{gathered}
\bar{w}=\frac{\sum_{i=1}^{k} w_{i}}{k} \\
s_{w}^{2}=\frac{1}{k-1}\left(\sum_{i=1}^{k} w_{i}^{2}-k \bar{w}^{2}\right) \\
U=(k-1)\left(\bar{w}-\frac{\hat{a} s_{w}^{2}}{k \bar{w}}\right)
\end{gathered}
$$

De los resultados (??), (??) y (??) se estima la varianza debida a la variación entre estudios obteniendo:

$$
\tau^{2}=\frac{Q-(k-1)}{U} \quad \text { cuando } Q>k-1
$$


donde $Q$ es el resultado de la prueba de heterogeneidad que se describe más adelante. Si $Q<k-1$ entonces la variación entre estudios se considera nula y el modelo coincide con el de efectos fijos descrito anteriormente.

Mediante la siguiente expresión se obtienen los pesos ajustados para cada estudio:

$$
w_{i}^{*}=\frac{1}{\frac{1}{w_{i}}+\tau^{2}}
$$

la estimación del efecto global y su respectiva varianza se muestran en (??) y (??):

$$
\begin{gathered}
\bar{y}=\frac{\sum_{i=1}^{k} w_{i}^{*} y_{i}}{\sum_{i=1}^{k} w_{i}^{*}} \\
V(\bar{y})=\frac{1}{\sum_{i=1}^{k} w_{i}^{*}}
\end{gathered}
$$

\section{Heterogeneidad}

A menudo ocurre que la variabilidad entre estudios es mayor a lo esperado por el azar [?] , y por ello es importante contrastar la hipótesis de homogeneidad. La prueba a utilizar es la desarrollada por Cochran en 1954, pero debido a su baja potencia, es recomendable verificar sus resultados por otros medios, como por ejemplo un análisis por subgrupos [?].

Esta prueba calcula la suma ponderada de las desviaciones al cuadrado del efecto determinado en cada estudio con respecto a la media global:

$$
Q=\sum_{i=1}^{k} w_{i}\left(y_{i}-\bar{y}\right)^{2}
$$

En esta prueba (??) de homogeneidad, $Q$ se distribuye asintóticamente como una $\chi^{2}$ con $k-1$ grados de libertad.

Se recomienda que aunque esta prueba muestre una heterogeneidad no significativa, se debe confirmar estos resultados con otras alternativas de tipo gráfico [?].

\subsection{Metanálisis Bayesiano}

En un enfoque Bayesiano se considera que todos los parámetros provienen de una superpoblación, con sus propios parámetros.

El metanálisis Bayesiano sintetiza los datos de los estudios realizados individualmente, incorporando la incertidumbre de los parámetros. Por otro lado, provee una distribución predictiva para futuras observaciones procedentes de cualquier estudio, que puede ser de fundamental interés para investigadores que deben tomar decisiones.

\section{Un modelo para efectos aleatorios}

Asumiéndose una estructura de error Gaussiana, se obtiene el siguiente modelo de efectos aleatorios:

$$
\begin{gathered}
y_{i} \sim N\left(\theta_{i}, \sigma_{i}^{2} / n_{i}\right) \\
\theta_{i} \sim N\left(\mu, \tau^{2}\right) \quad i=1,2, \ldots, k
\end{gathered}
$$


En el modelo (??), $\theta_{i}$ es el verdadero, pero desconocido, efecto en el $i$-ésimo estudio, y $\mu$ es el efecto poblacional también desconocido, y de mayor interés, ya que representa el efecto combinado obtenido de los resultados individuales. Adicionalmente, $\tau^{2}$ es la varianza poblacional, que cuantifica la dispersión entre estudios, la cual informa de cuán variable es este efecto en la población. En el caso de que $\tau^{2}=0$ se obtiene el modelo dado en la Sección 2.1.1.

En un enfoque Bayesiano se desearía conocer distribuciones a priori sobre los parámetros desconocidos de (??), esto es, $\sigma_{i}^{2} \mathrm{~s}, \mu \mathrm{y} \tau^{2}$. En la práctica esta información a priori probablemente esté disponible para $\mu$, el efecto poblacional, y $\tau^{2}$ la variabilidad poblacional del efecto sobre los estudios. Por tanto para $\sigma_{i}^{2}$ , las varianzas de los resultados individuales, se asume un conjunto no informativo de distribuciones a priori [?].

Siguiendo el proceso de inferencia Bayesiana tradicional, se utiliza el método de Jeffrey para obtener distribuciones a priori proporcionales a la inversa de las varianzas individuales, esto es, $P\left(\sigma_{i}^{2}\right) \propto 1 / \sigma_{i}^{2}$. Se ha observado que el uso de esta distribución a priori proporciona resultados muy similares a los obtenidos por la presunción común que $\operatorname{los} \sigma_{i}^{2}$ son conocidos y reemplazados por $\operatorname{los} s_{i}^{2}$, las varianzas observadas dentro de los estudios.

La distribución a priori para $\tau^{2}$ debe de ser lo suficientemente flexible para poder incorporar fácilmente información a priori mientras que al mismo tiempo sea matemáticamente tratable. Por este motivo la distribución gamma inversa es utilizada [?]. Para los propósitos de este trabajo se asume solamente información a priori no informativa para el efecto poblacional $\mu$, manteniéndose algo de objetividad con respecto a la estimación del efecto global. Se asume que esta sigue una distribución a priori localmente uniforme.

\section{Método de Laplace}

Una de las dificultades del enfoque Bayesiano es el obtener estimaciones posteriores precisas sin aumentar en demasía la dificultad matemática. Una técnica basada en la expansión en serie de potencias que se utiliza para este fin en conocida como el Método de Laplace. Este método se basa en suponer una función $f$ que depende del vector de parámetros $m$-dimensional $\theta$, suave y positiva. Además se tiene la función $h$ de $\boldsymbol{\theta}$, donde $-h$ tiene un único máximo en $\widehat{\boldsymbol{\theta}}$. Sin pérdida de generalidad, se toma $\theta$ univariado, y se desea aproximar la siguiente integral:

$$
I=\int f(\theta) e^{-n h(\theta)} d \theta
$$

Expandiendo a $f$ y $h$ en series de Taylor en (??) alrededor de $\widehat{\theta}$, se obtiene:

$$
\begin{gathered}
I=\int\left[f(\widehat{\theta})+\frac{f^{\prime}(\widehat{\theta})}{1 !}(\theta-\widehat{\theta})+\frac{f^{\prime \prime}(\widehat{\theta})}{2 !}(\theta-\widehat{\theta})^{2}\right] \\
\times \exp \left[-n h(\theta)-n h^{\prime}(\theta)(\theta-\widehat{\theta})-n h^{\prime \prime}(\widehat{\theta}) \frac{(\theta-\widehat{\theta})^{2}}{2 !}\right] d \theta \\
=e^{-n h(\widehat{\theta})} \int\left[f(\widehat{\theta})+f^{\prime}(\widehat{\theta})(\theta-\widehat{\theta})+\frac{f^{\prime \prime}(\widehat{\theta})}{2}(\theta-\widehat{\theta})^{2}\right] \\
\times \exp \left[-\frac{(\theta-\widehat{\theta})^{2}}{2\left(n h^{\prime \prime}(\widehat{\theta})\right)^{-1}}\right] d \theta
\end{gathered}
$$

desde que $h^{\prime}(\widehat{\theta})=0$ por definición de $\widehat{\theta}$. Se observa que el término final dentro de la integral (??) es proporcional a una densidad $N\left(\widehat{\theta},\left[n h^{\prime \prime}(\widehat{\theta})\right]^{-1}\right)$ para $\theta$, además el segundo término dentro de esta integral desaparece ya que $E(\theta-\widehat{\theta})=0$. De esta manera se obtiene la siguiente aproximación:

$$
I \approx e^{-n h(\widehat{\theta})} f(\widehat{\theta}) \sqrt{\frac{2 \pi}{n h^{\prime \prime}(\widehat{\theta})}}\left(1+\frac{f^{\prime \prime}(\widehat{\theta})}{2 f(\widehat{\theta})} V(\theta)\right)
$$


donde $V(\theta)=\left[n h^{\prime \prime}(\widehat{\theta})\right]^{-1}=O\left(\frac{1}{n}\right)$. En general, para el caso del vector de parámetros $\theta$, se tiene que $I=\widehat{I}\left\{1+O\left(\frac{1}{n}\right)\right\}$, donde:

$$
\widehat{I}=f(\widehat{\boldsymbol{\theta}})\left(\frac{2 \pi}{n}\right)^{m / 2}|\tilde{\Sigma}|^{1 / 2} \exp (-n h(\widehat{\boldsymbol{\theta}}))
$$

y $\tilde{\Sigma}=\left[D^{2} h(\widehat{\boldsymbol{\theta}})\right]^{-1}$. Entonces, (??) es una aproximación de primer orden de (??) en el sentido asintótico. Ahora, el interés se orienta a obtener el valor esperado posterior de una función $g(\boldsymbol{\theta})$, donde $-n h(\boldsymbol{\theta})$ es vista como una densidad posterior no normalizada, $\log [f(\mathbf{y} \mid \boldsymbol{\theta}) \pi(\boldsymbol{\theta})]$. Entonces:

$$
E(g(\boldsymbol{\theta}))=\frac{\int g(\boldsymbol{\theta}) \exp (-n h(\widehat{\boldsymbol{\theta}})) d \boldsymbol{\theta}}{\int \exp (-n h(\widehat{\boldsymbol{\theta}})) d \boldsymbol{\theta}}
$$

Para evaluar la integral (??) se aplica el método de Laplace (??) haciendo para el numerador $f=g$ y denominador $f=1$ obteniéndose:

$$
\begin{aligned}
E(g(\boldsymbol{\theta})) & =\frac{g(\widehat{\boldsymbol{\theta}})\left(\frac{2 \pi}{n}\right)^{m / 2}|\tilde{\Sigma}|^{1 / 2} \exp (-n h(\widehat{\boldsymbol{\theta}}))\left[1+O_{1}\left(\frac{1}{n}\right)\right]}{\left(\frac{2 \pi}{n}\right)^{m / 2}|\tilde{\Sigma}|^{1 / 2} \exp (-n h(\widehat{\boldsymbol{\theta}}))\left[1+O_{2}\left(\frac{1}{n}\right)\right]} \\
& =g(\widehat{\boldsymbol{\theta}})\left[\frac{1+O_{2}\left(\frac{1}{n}\right)-O_{2}\left(\frac{1}{n}\right)+O_{1}\left(\frac{1}{n}\right)}{1+O_{2}\left(\frac{1}{n}\right)}\right] \\
= & g(\widehat{\boldsymbol{\theta}})\left[1+\frac{O_{3}\left(\frac{1}{n}\right)}{1+O_{2}\left(\frac{1}{n}\right)}\right]=g(\widehat{\boldsymbol{\theta}})\left[1+O\left(\frac{1}{n}\right)\right]
\end{aligned}
$$

mostrando que $g(\widehat{\boldsymbol{\theta}})$ es una aproximación de primer orden para la media posterior de $g(\boldsymbol{\theta})$.

\section{Inferencia aproximada}

La base de las aproximaciones para el primer y segundo momento posterior de los parámetros en el modelo (??) es la aproximación de la densidad marginal de los datos, $m(y)$

$$
\begin{aligned}
& m(y) \propto \int_{\mathbb{R}^{k}} \int_{0}^{+\infty} \ldots \int_{0}^{+\infty} \int_{0}^{+\infty} \int_{-\infty}^{+\infty} \prod_{i=1}^{k}\left(\frac{1}{\sigma_{i}^{2}}\right)^{\left(n_{i}+2\right) / 2}\left(\frac{1}{2 \pi}\right)^{n_{i} / 2} \\
& \times \exp \left\{-\frac{n_{i} s_{i}^{2}}{2 \sigma_{i}^{2}}-\frac{n_{i}\left(y_{i}-\theta_{i}\right)^{2}}{2 \sigma_{i}^{2}}\right\} N_{k}\left(\boldsymbol{\theta} \mid \mu, \tau^{2} \mathbf{I}\right) I G\left(\tau^{2} \mid a, b\right) d \mu d \tau^{2} d \sigma^{2} d \boldsymbol{\theta}
\end{aligned}
$$

donde $s_{i}^{2}$ es la varianza observada del $i$-ésimo resultado, $N_{k}(\mathbf{x} \mid \nu, \mathbf{W})$ denota una densidad Normal $k$ variada evaluada en $\mathbf{x}$, con vector de medias $\nu$ y matriz de varianza-covarianza $\mathbf{W} ; I G(x \mid \alpha, \beta)$ denota una densidad gamma inversa evaluada en $x$, con parámetros $\alpha$ y $\beta$ e I es una matriz identidad de orden $k$. Cambiando el orden de integración e integrando sobre $\boldsymbol{\theta}$ en (??), se obtiene:

$$
\begin{aligned}
& m(y)=\int_{0}^{+\infty} \cdots \int_{0}^{+\infty} \int_{0}^{+\infty} \int_{-\infty}^{+\infty} \prod_{i=1}^{k}\left(\frac{2 \pi}{n_{i}}\right)^{1 / 2} \prod_{i=1}^{k}\left(\frac{1}{\sigma_{i}^{2}}\right)^{\left(n_{i}+1\right) / 2}\left(\frac{1}{2 \pi}\right)^{n_{i} / 2} \\
& \times N_{k}\left(\mathbf{y} \mid \mu, \operatorname{diag}\left(\sigma_{i}^{2} / n_{i}\right)+\tau^{2} \mathbf{I}\right) I G\left(\tau^{2} \mid a, b\right) \exp \left\{-\sum n_{i} s_{i}^{2} / 2 \sigma_{i}^{2}\right\} d \mu d \tau^{2} d \sigma^{2}
\end{aligned}
$$


donde $\mathbf{y}=\left(y_{1}, \ldots, y_{k}\right)^{T}$. Para grandes $n_{i}, N_{k}\left(\mathbf{y} \mid \mu, \operatorname{diag}\left(\sigma_{i}^{2} / n_{i}\right)+\tau^{2} \mathbf{I}\right)$ tiende a $N_{k}\left(\mathbf{y} \mid \mu, \tau^{2} \mathbf{I}\right)$. Todo lo dicho anteriormente produce la siguiente aproximación para $m(y)$ :

$$
\begin{aligned}
m(y)= & \int_{0}^{+\infty} \ldots \int_{0}^{+\infty} \int_{0}^{+\infty} \int_{-\infty}^{+\infty} \prod_{i=1}^{k}\left(\frac{2 \pi}{n_{i}}\right)^{1 / 2} \prod_{i=1}^{k}\left(\frac{1}{\sigma_{i}^{2}}\right)^{\left(n_{i}+1\right) / 2}\left(\frac{1}{2 \pi}\right)^{n_{i} / 2} \\
& \times N_{k}\left(\mathbf{y} \mid \mu, \tau^{2} \mathbf{I}\right) I G\left(\tau^{2} \mid a, b\right) \exp \left\{-\sum n_{i} s_{i}^{2} / 2 \sigma_{i}^{2}\right\} d \mu d \tau^{2} d \sigma^{2}
\end{aligned}
$$

A partir de (??) se tiene el primer momento del efecto del $i$-Ã@simo estudio como se describió en el modelo (??) y que está dado por:

$$
E\left(\theta_{i} \mid \mathbf{y}, \mathbf{s}^{2}, \mathbf{n}\right) \approx \bar{y}-\frac{\left(n_{i}-1\right) s_{i}^{2} b(2 a+k-1)}{2 n_{i}\left(n_{i}-3\right)\left(1+b \mathbf{R S S}_{\mathbf{B}} / 2\right)}\left(y_{i}-\bar{y}\right)
$$

donde $\mathbf{y}$ se definió anteriormente, $\mathbf{s}^{2}=\left(s_{1}^{2}, \ldots, s_{k}^{2}\right)^{T}$, es el vector varianzas observadas de cada estudio, $\mathbf{n}=\left(n_{1}, \ldots, n_{k}\right)^{T}$ el vector de los tamaños muestrales de cada uno de los estudios y $\mathbf{R S S}_{\mathbf{B}}=\sum_{i=1}^{k} y_{i}^{2}-k \bar{y}^{2}$ es la suma de cuadrados residual entre estudios. También, $\bar{y}$ es la media aritmética de los efectos individuales $y_{i}, a$ y $b$ son los parámetros de la distribución a priori gamma inversa para $\tau^{2}$. Una aproximación para el segundo momento está dado por:

$$
\begin{aligned}
V\left(\theta_{i} \mid \mathbf{y}, \mathbf{s}^{2}, \mathbf{n}\right) \approx \frac{\left(n_{i}-1\right)}{n_{i}\left(n_{i}-3\right)} & \left\{1+\frac{\left(n_{i}-1\right) s_{i}^{2} b^{2}(2 a+k-1)\left(n_{i}-4+2 a+k\right)\left(y_{i}-\bar{y}\right)^{2}}{2 n_{i}\left(n_{i}-3\right)\left(n_{i}-5\right)\left(1+b \mathbf{R S S}_{\mathbf{B}} / 2\right)^{2}}\right. \\
+ & \left.\frac{\left(n_{i}-1\right) s_{i}^{2} b^{2}(2 a+k-1)}{2 k n_{i}\left(n_{i}-5\right)\left(1+b \mathbf{R S S}_{\mathbf{B}} / 2\right)}\right\}
\end{aligned}
$$

Similarmente, una aproximación para el primer momento de $\sigma_{i}^{2}$, la variabilidad en el $i$-ésimo estudio, está dada por:

$$
E\left(\sigma_{i}^{2} \mid \mathbf{s}^{2}, \mathbf{n}\right) \approx \frac{n_{i}}{n_{i}-2} s_{i}^{2}
$$

Considerando el efecto poblacional, $\mu$, aproximaciones iniciales, basadas en las expansiones de Taylor de primer orden, para el primer y segundo momento están dadas por:

$$
E\left(\mu \mid \mathbf{y}, \mathbf{s}^{2}, \mathbf{n}\right) \approx \bar{y}, V\left(\mu \mid \mathbf{y}, \mathbf{s}^{2}, \mathbf{n}\right) \approx \frac{2\left(1+b \mathbf{R S S}_{\mathbf{B}} / 2\right)}{b k(2 a+k-3)}
$$

Finalmente, aproximaciones para el primer y segundo momento de la variabilidad poblacional, $\tau^{2}$, están dadas por:

$$
E\left(\tau^{2} \mid \mathbf{y}, \mathbf{s}^{2}, \mathbf{n}\right) \approx \frac{2\left(1+b \mathbf{R S S}_{\mathbf{B}} / 2\right)}{b(2 a+k-3)}, V\left(\tau^{2} \mid \mathbf{y}, \mathbf{s}^{2}, \mathbf{n}\right) \approx \frac{8\left(1+b \mathbf{R S S}_{\mathbf{B}} / 2\right)^{2}}{b^{2}(2 a+k-3)^{2}(k+2 a-5)}
$$

Cabe señalar que todas las aproximaciones tratadas en esta sección, son obtenidas utilizando expansiones de Taylor de primer orden de la densidad posterior.

\section{Resultados}

\subsection{Metanálisis Clásico}

Para obtener los resultados presentados en este artículo, se utilizó la base de datos de las RMM de los países de Sudamérica y Latinoamérica de los años 1990, 1995, 2000, 2005 y 2008, publicadas por la OMS [?].

Para obtener los errores estándar de las estimaciones individuales, los datos se transformaron a escala logarítmica y se extrajo los errores a partir de los intervalos de confianza (la base de datos sólo contiene la estimación puntual y su respectivo intervalo de confianza al $95 \%$, más no el error estándar).

Para combinar las diferentes RMM de los países de Sudamérica y Latinoamérica, se aplicó el modelo de efectos fijos (no heterogeneidad) y luego, el modelo de efectos aleatorios (heterogeneidad). Previamente se utilizó la prueba Q para contrastar la siguiente hipótesis de homogeneidad: 
1. $H_{0}$ : La RMM es homogénea entre los países de Sudamérica.

$H_{1}$ : La RMM no es homogénea entre los países de Sudamérica.

2. $H_{0}$ : La RMM es homogénea entre los países de Latinoamérica.

$H_{1}$ : La RMM no es homogénea entre los países de Latinoamérica.

A la luz de los resultados presentados en las tablas 1 y 2 , se determinó que:

1. La RMM es heterogénea entre los países sudamericanos.

2. La RMM es heterogénea entre los países latinoamericanos.

Por tanto se decidió aplicar el modelo de efectos aleatorios para combinar las RMM de los países de la región.

Los siguientes gráficos y tablas se obtuvieron como resultado de la elaboración y ejecución de los códigos fuente respectivos en el software $\mathrm{R}$ Project:

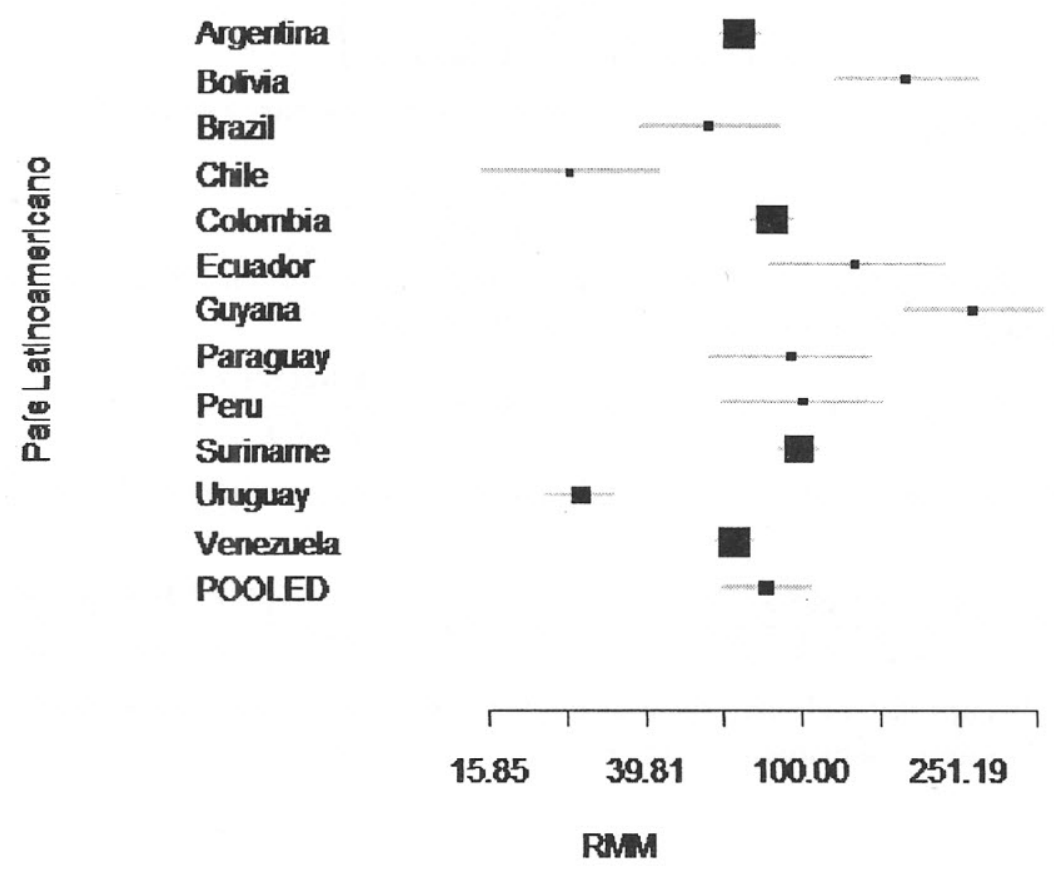

Figura 2: Metaplot de las RMM en Sudamérica 2008

\begin{tabular}{|c|c|c|c|c|c|c|}
\hline \multicolumn{7}{|c|}{ Tabla 1. Razón de Mortalidad Materna SUDAMÉRICA } \\
\hline Año & Prueba Q & p-value & Modelo efectos & RMM pooled & LI (95\%) & LS (95\%) \\
\hline 1990 & 0.25 & 0 & alcatorios & 123.0 & 92.2 & 165.0 \\
\hline 1995 & 0.33 & 0 & aleatorios & 101.0 & 71.5 & 142.0 \\
\hline 2000 & 0.26 & 0 & aleatorios & 90.1 & 66.1 & 123.0 \\
\hline 2005 & 0.17 & 0 & aleatorios & 82.1 & 63.5 & 106.0 \\
\hline 2008 & 0.18 & 0 & alcatorios & 80.7 & 62.1 & 105.0 \\
\hline
\end{tabular}




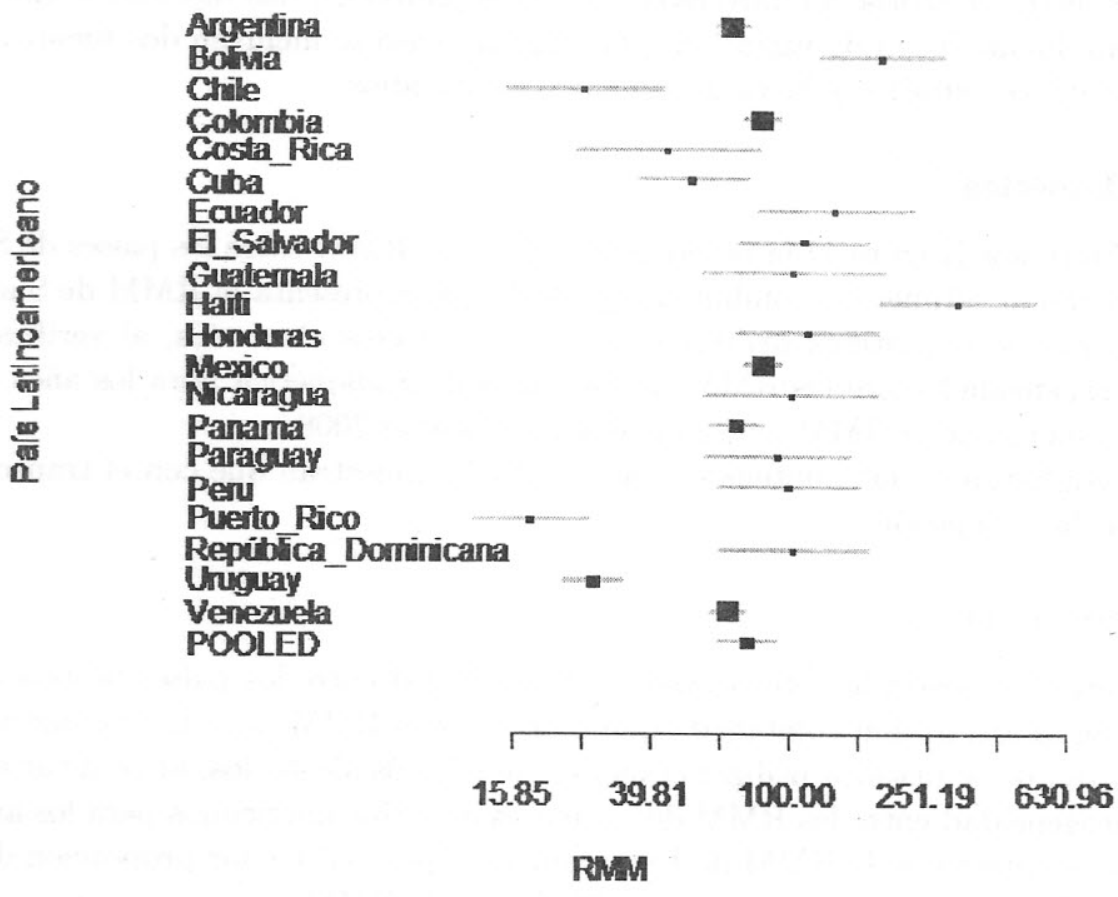

Figura 3: Metaplot de las RMM en Latinoamérica 2008

\begin{tabular}{|c|c|c|c|c|c|c|}
\hline \multicolumn{7}{|c|}{ Tabla 2. Razón de Mortalidad Materna LATINOAMÉRICA } \\
\hline Año & Prucba Q & p-value & Modelo efectos & MMR pooled & LI (95\%) & LS (95\%) \\
\hline 1990 & 0.25 & 0 & aleatorios & 117.0 & 92.3 & 149.0 \\
\hline 1995 & 0.24 & 0 & aleatorios & 105.0 & 82.8 & 132.0 \\
\hline 2000 & 0.25 & 0 & aleatorios & 90.1 & 71.0 & 114.0 \\
\hline 2005 & 0.17 & 0 & aleatorios & 79.1 & 64.4 & 97.0 \\
\hline 2008 & 0.17 & 0 & aleatorios & 75.5 & 61.7 & 92.5 \\
\hline
\end{tabular}

\subsection{Metanálisis Bayesiano}

Como la base de datos del IDH [?] sólo se dispone de la estimación puntual; para poder combinar estas razones de los países de la región se utilizó el metanálisis Bayesiano con una distribución gamma inversa a priori, con parámetros $a=0$ y $b=2$, recomendada cuando no se dispone de mayor información o la que se dispone es vaga [?].

Al no tener información del tamaño de muestra, error estándar entre otros, se consideró apropiado aplicar el metanálisis Bayesiano para obtener el IDH de la región.

Los resultados que se obtuvieron después de elaborar los códigos fuentes respectivos en MATLAB y luego de ejecutarlos se muestran en la Tabla 3 :

\begin{tabular}{|c|c|c|c|c|c|c|}
\hline \multicolumn{7}{|c|}{ Tabla 3. Índice de Desarrollo Humano } \\
\hline Región & $\mathrm{E}(\mu)$ & $\mathrm{V}(\mu)$ & $\mathrm{LI}(\mu) 95 \%$ & $\mathrm{LS}(\mu) 95 \%$ & $\mathrm{E}\left(\mathrm{r}^{2}\right)$ & $\mathrm{V}\left(\mathrm{r}^{2}\right)$ \\
\hline SUDAMÉRICA & 0.6971 & 0.0096 & 0.5052 & 0.8890 & 0.1150 & 0.0038 \\
\hline LATINOAMÉRICA & 0.6719 & 0.0043 & 0.5438 & 08000 & 0.0769 & 0.0009 \\
\hline
\end{tabular}




\section{Discusión}

\subsection{Metanálisis Clásico}

En el modelo de efectos aleatorios la inferencia se basa en suponer que los estudios incluidos en el análisis constituyen una muestra aleatoria del universo de estudios posibles, y sus resultados son conservadores al tener en cuenta una fuente extra de variación, para nuestro caso se incluyen dos fuentes de variabilidad: la existente dentro de los estudios y la variación entre los estudios.

\section{RMM para Sudamérica}

Se muestra en el Metaplot (Figura 1) la heterogeneidad de las RMM entre los países de Sudamérica para el año 2008, junto con la estimación combinada (pooled) que representa la RMM de Sudamérica.

La Tabla 1, muestra que se justifica utilizar el modelo de efectos aleatorios, al verificar con la prueba Q que existe heterogeneidad entre las RMM de los países de Sudamérica para los años bajo estudio. Se observa un decrecimiento de la RMM en Sudamérica de 1990 al 2008.

Los intervalos de confianza al $95 \%$ obtenidos para la RMM, muestran que con el transcurso de los años disminuye el error de estimación.

\section{RMM para Latinoamérica}

El Metaplot (Figura 2) muestra la heterogeneidad de las RMM entre los países latinoamericanos para el año 2008, junto con la estimación combinada que representa el RMM para Latinoamérica.

La Tabla 2, muestra que se justifica utilizar el modelo de efectos aleatorios, al verificar con la estadística Q que existe heterogeneidad entre las RMM de los países de latinoamericanos para los años bajo estudio. Se observa un decrecimiento de la RMM de Latinoamérica, pero en menor proporción del 2005 al 2008. Se muestra además los intervalos de confianza al $95 \%$ para la RMM.

\subsection{Metanálisis Bayesiano}

Es una metodología con una fuerte base estadística que permite obtener la distribución completa de las medidas de interés teniendo en cuenta la incertidumbre inherente al proceso.

En la Tabla 3 se muestran los resultados de los estudios de Sudamérica y Latinoamérica. De esta tabla se observa que el IDH de los países de Sudamérica es mayor al de Latinoamérica. Adicionalmente, se muestran estimaciones por intervalos al $95 \%$ de confianza para las regiones.

\section{Conclusiones}

1. El metaanálisis utilizado en el presente artículo es un estudio basado en la integración estructurada y sistemática de la información obtenida sobre las RMM de los países sudamericanos y latinoamericanos para los años 1990, 1995, 2000, 2005 y 2008, con la finalidad de obtener una estimación cuantitativa sintética, que se muestra en la Tabla 4.

\begin{tabular}{|c|c|c|}
\hline \multicolumn{3}{|c|}{ Tabla 4. Razón de Mortalidad Materna } \\
\hline Año & SUDAMÉRICA & LATINOAMÉRICA \\
\hline 1990 & 123.0 & 117.0 \\
\hline 1995 & 101.0 & 105.0 \\
\hline 2000 & 90.1 & 90.1 \\
\hline 2005 & 82.1 & 79.1 \\
\hline 2008 & 80.7 & 75.5 \\
\hline
\end{tabular}

2. Para trabajar las bases de datos, previamente se transformaron estas razones a escala logarítmica y luego se obtuvo el error estándar de la estimación, despejándolo del intervalo de confianza puesto que dicha base publicada por la OMS sólo contiene la estimación puntual y su respectivo intervalo 
de confianza al $95 \%$ para cada país de la región. El resumen de los resultados se presenta en la Tabla 4.

3. El metaanálisis Bayesiano aplicado en la presente investigación es un método basado en la integración ordenada y lógica de la información obtenida del IDH de los países de Sudamérica y Latinoamérica para el año 2010 incorporando la incertidumbre de los parámetros, con la finalidad de obtener una estimación cuantitativa sintética, la que se muestra en la Tabla 5.

\begin{tabular}{|c|c|}
\hline \multicolumn{2}{|c|}{ Tabla 5. Índice de Desarrollo Humano } \\
\hline Región & 2010 \\
\hline SUDAMÉRICA & 0.6971 \\
\hline LATINOAMÉRICA & 0.6719 \\
\hline
\end{tabular}

4. Como la base de datos del IDH publicado por el PNUD sólo tiene el estimador puntual, para combinar los IDH de los países de Sudamérica y Latinoamérica se utilizó el metanálisis Bayesiano con una distribución gamma inversa a priori con parámetros $a=0, b=2$.

5. Esta forma de obtener estimaciones cuantitativas sintéticas en el presente artículo, es una propuesta para estimar la RMM y el IDH para las regiones de Sudamérica y Latinoamérica, la cual se puede extender a otras regiones. 


\section{Bibliografía}

[1] ABRAMS, K.; SANSÓ, B. Approximate Bayesian inference for random effects meta-analysis. Rev. Statistics in medicine, vol. 17, p. 201-218, 1998.

[2] CARLIN, B.; LOUIS, T. Bayes and empirical Bayes methods for data analysis. New York: Chapman \& Hall/CRC, 2000.

[3] HARTUNG, J.; KNAPP, G.; SINHA, B. Statistical meta-analysis with applications. New Jersey: John Wiley \& Sons, Inc.; 2008.

[4] HOBBS, B. Bayesian meta-analysis with hierarchical modeling. Minneapolis: Division of Biostatistics, School of Public Health, University of Minnesota; 2008.

[5] MOLINERO, L. Heterogeneidad entre los estudios incluidos en un meta-análisis. España: Asociación de la Sociedad Española de Hipertensión Liga Española para la lucha contra la Hipertensión Arterial; 2003.

[6] MOLINERO, L. Meta-análisis. España: Asociación de la Sociedad Española de Hipertensión Liga Española para la lucha contra la Hipertensión Arterial; 2003.

[7] MORAL, E. Predicción meta-analítica a la Bayesiana para la valoración de bienes que no tienen mercado. Documento de trabajo de la evaluación económica de proyectos de transporte. España: CEDEX; 2009.

[8] OMS. Mortalidad Materna 2005. Estimaciones elaboradas por la OMS, el UNICEF, el UNFPA y el Banco Mundial.; 2008.

[9] PNUD. Informe Regional sobre Desarrollo Humano para América Latina y el Caribe 2010. Costa Rica: Editorama, S.A.; 2010.

[10] PNUD. Informe sobre Desarrollo Humano 2010. España: Ediciones Mundi-Prensa; 2010. 